\title{
Brain-Specific SNAP-25 Deletion Leads to Elevated Extracellular Glutamate Level and Schizophrenia-Like Behavior in Mice
}

\author{
Hua Yang, ${ }^{1}$ Mengjie Zhang, Jiahao Shi, ${ }^{1}$ Yunhe Zhou, ${ }^{1}$ Zhipeng Wan, ${ }^{1}$ Yicheng Wang, \\ Yinghan Wan, ${ }^{2}$ Jun $\mathrm{Li}^{2}{ }^{2}$ Zhugang Wang, ${ }^{2}$ and Jian Fei ${ }^{1,2}$ \\ ${ }^{1}$ School of Life Science and Technology, Tongji University, Shanghai 200092, China \\ ${ }^{2}$ Shanghai Engineering Research Center of Model Organisms (SRCMO/SMOC), Shanghai 201203, China \\ Correspondence should be addressed to Jian Fei; jfei@tongji.edu.cn
}

Received 29 June 2017; Revised 31 August 2017; Accepted 15 October 2017; Published 28 November 2017

Academic Editor: Depei Li

Copyright (c) 2017 Hua Yang et al. This is an open access article distributed under the Creative Commons Attribution License, which permits unrestricted use, distribution, and reproduction in any medium, provided the original work is properly cited.

\begin{abstract}
Several studies have associated reduced expression of synaptosomal-associated protein of $25 \mathrm{kDa}$ (SNAP-25) with schizophrenia, yet little is known about its role in the illness. In this paper, a forebrain glutamatergic neuron-specific SNAP-25 knockout mouse model was constructed and studied to explore the possible pathogenetic role of SNAP-25 in schizophrenia. We showed that SNAP-25 conditional knockout (cKO) mice exhibited typical schizophrenia-like phenotype. A significantly elevated extracellular glutamate level was detected in the cerebral cortex of the mouse model. Compared with Ctrls, SNAP-25 was dramatically reduced by about $60 \%$ both in cytoplasm and in membrane fractions of cerebral cortex of cKOs, while the other two core members of SNARE complex: Syntaxin-1 (increased 80\%) and Vamp2 (increased 96\%) were significantly increased in cell membrane part. Riluzole, a glutamate release inhibitor, significantly attenuated the locomotor hyperactivity deficits in cKO mice. Our findings provide in vivo functional evidence showing a critical role of SNAP-25 dysfunction on synaptic transmission, which contributes to the developmental of schizophrenia. It is suggested that a SNAP-25 cKO mouse, a valuable model for schizophrenia, could address questions regarding presynaptic alterations that contribute to the etiopathophysiology of SZ and help to consummate the pre- and postsynaptic glutamatergic pathogenesis of the illness.
\end{abstract}

\section{Introduction}

Schizophrenia (SZ), a complicated psychiatric disorder, affects almost 1 percent of the general population in the world $[1,2]$. While the etiology and pathophysiology of SZ remain elusive, genetic risk factors are recognized as an important contributing factor to the pathogenesis of this neuropsychiatric disorder [3]. It has been documented that the synaptosomal-associated protein of $25 \mathrm{kDa}$ (SNAP-25) is a candidate risk gene for $\mathrm{SZ}$, as supported by the following lines of evidence: (1) Genetic association and linkage studies have revealed that chromosome region 20p12.2 which SNAP-25 locates in has significant linkage with SZ $[4,5]$. (2) Large-scale genome-associated case-control studies have revealed that several single nucleotide polymorphisms (SNPs) of SNAP-25 are significantly associated with SZ [6]. (3) Various postmortem analyses have found that the expression of SNAP-25 is reduced in prefrontal cortex (PFC) and hippocampus in brain of patients with SZ [7-9]. However, how the reduction of SNAP-25 level is involved in the pathological phenotype remains unknown.

In the brain, SNAP-25 proteins are abundantly expressed in glutamatergic terminals, while relatively lower amounts of the protein are detectable in GABAergic terminals [10]. The primary role of SNAP-25 is a fundamental component of soluble N-ethylmaleimide-sensitive factor attachment protein receptor (SNARE). Together with cell membrane protein syntaxin and vesicle-associated membrane protein (VAMP), SNAP-25 mediates presynaptic vesicle docking and exocytosis [11]. In addition to regulation of synaptic transmission, SNAP-25 is also believed to regulate intracellular calcium dynamics through negative modulation of voltage-gated calcium channels. It also plays a role in other neuronal processes, including spine morphogenesis, postsynaptic receptor 
trafficking, and neuronal plasticity [12]. But the underlying cellular mechanisms still need to be explored.

Over the past few decades, several mouse models have been constructed to elucidate the physiological role of SNAP-25 in vivo. Complete knockout of SNAP-25 in mice leads to no evoked exocytosis and death of the animal at birth. However, heterozygous mice are able to survive and exhibit locomotor hyperactivity and learning deficiencies. SNAP-25 knock-in mice, which have a single amino acid substitution of Ala for Ser187, have been shown to display epilepsy and anxiety-related behavior. The blind-drunk (Bdr) mouse expresses a dominant point mutant SNAP-25b protein, resulting in impaired sensorimotor gating and ataxia, while SNAP-25b-deficient model has developmental defects, seizures, and impaired synaptic plasticity. Conclusively, SNAP-25 mouse mutants occupied a series of psychophenotypes. However, the results derived from different models were inconsistent or conflicting, and no convincing evidence has supported an association between SNAP-25 and SZ. Therefore, additional investigations are necessary to demonstrate the possible role of SNAP-25 in SZ.

Taking into account that the cerebral cortex and hippocampus are the critical brain areas associated with SZ, brain-specific SNAP-25 knockout mice are most suitable to explore the relationship between SNAP-25 and SZ. Furthermore, SNAP-25 has been highly expressed both in neuron and endocrine cells, so brain-targeted SNAP-25 modification is capable of excluding interference signals from the surrounding system. In this study, we designed a brainspecific SNAP-25 knockout mouse, through behavioral phenotyping, molecular detection, and drug treatment within this model, to explore the possible pathogenetic role of SNAP-25 in SZ.

\section{Materials and Methods}

2.1. Animals. Mice were maintained in a specific pathogenfree (SPF) facility under a $12 \mathrm{~h}$ light/dark cycles. All animal protocols were approved by the Institutional Animal Care and Use Committee at Shanghai Research Center for Model Organisms (number 2015-0005). Mice were sacrificed with carbon dioxide when experiments were completed.

2.2. Generation of SNAP-25 cKO Mice. Genomic DNA of SCR012 ES cells isolated from 129 S6/SvEv mouse strain was utilized to amplify SNAP-25 homologous fragments. The targeting strategy is flanking exon 4 of the mouse SNAP-25 gene with two loxP cassettes. Chimera mice were constructed by injecting recombination-positive ES cells into blastocyst and backcrossed to C57BL/6J mice. SNAP-25 ${ }^{\mathrm{L} 2 / \mathrm{L} 2}$ : CaMKII $\alpha-\mathrm{Cre}^{+/ \mathrm{wt}}$ mice were obtained by regular breeding procedure between SNAP-25 $5^{\mathrm{L} / \mathrm{L} 2}$ mice and CaMKII $\alpha$-Cre strain (The Jackson Laboratory, stock number 005359) in which Cre-recombinase is extensively expressed in forebrain excitatory neurons by $\mathrm{p} 20[13,14]$. PCR genotyping was performed with genomic DNA isolated from mouse tail tissue. The primers used for genotyping were designed for identifying loxP (forward: $5^{\prime}$-CACTGCAGAGATTGCA
GTATCACTA-3', reverse: $5^{\prime}$ - CAATGCACAGTTATTG TATTGAAGG- $3^{\prime}$ ), and Cre sequences (forward: $5^{\prime}$ - AGCG ATGGATTTCCGTCTCTGG-3 ${ }^{\prime}$, reverse: $5^{\prime}$ - AGCTTGCAT GATCTCCGGTATTGAA - $3^{\prime}$ ).

2.3. Western Blot Analysis. Cell cytosolic or membrane protein lysates of mouse brain tissues were prepared using Mem-PER Plus Membrane Protein Extraction Kit (Thermo Scientific, 89842). Then, the lysates were separated by SDSPAGE and probed with specific antibodies: SNAP-25 (Abcam, ab66066), SNAP-23 (Abcam, ab3340), syntaxin (Santa Cruz, sc-12736), Vamp2 (Abcam, ab6276), Munc-18 (SYSY, 116002), Phospho-Synaptotagmin (R\&D Systems, PPS085), $\beta$-ACTIN (Abcam, ab6276), TUBULIN (Abcam, ab15246), and $\mathrm{Na} / \mathrm{K}$ ATPase (Millipore, 05-369). For quantification, the densitometry measurement of each band was first normalized to that of $\beta$-ACTIN, TUBULIN, or $\mathrm{Na} / \mathrm{K}$ ATPase (used as loading control) and then averaged from at least three independent samples.

2.4. Immunofluorescence Staining. Sagittal brain sections ( $15 \mu \mathrm{m}$ in thickness) were prepared from a fixed brain with $4 \%$ paraformaldehyde, and immunostaining was performed as described [15]. Antibodies used for the immunostaining were SNAP-25 (Abcam, ab66066) and VGLUT1 (SYSY, 135304). Fluorescence was analyzed on a Nikon A1R confocal microscope (Nikon Instruments, Shanghai, CN).

2.5. Behavioral Testing. Behavioral phenotyping was performed on age-paired adult male mice (8 to 12 weeks for both $\mathrm{cKO}$ and Ctrl littermates). Prior to testing, mice were habituated to the testing room for $2 \mathrm{~h}$.

2.5.1. Open-Field Test. The open field is a square arena $(40 \times 42 \times 30 \mathrm{~cm}) .8 \mathrm{~cm}$ width elongated area along the wall is defined as the "peripheral zone," approximately $66 \%$ of the total area. We place the mouse in the center of the box and enable it to move freely for 15 mins, and an infrared tracking system (Kinder Scientific, Julian, USA) was borrowed to record the movement.

2.5.2. Prepulse Inhibition of the Startle Response. The Acoustic Startle Reflex Starter Package and Startle Reflex 5 software system (Med Associates Inc., St. Albans, VT) was used to assess prepulse inhibition (PPI). The test began with placing the mouse in the chamber's cylinder to acclimate for 5 mins. The remainder of the test consisted of two blocks of trials. A $65 \mathrm{~dB}$ background sound was presented throughout the session. The first block consisted of twenty trials of $20 \mathrm{~ms}$ and $105 \mathrm{~dB}$ sound served as startle stimuli and presented with different intertrial intervals (10-30s). The second block consisted of 50 trials, with five varying trial types: startle only, or a $10 \mathrm{~ms}$ prepulse sound at $70,75,80$, and $85 \mathrm{~dB}$ appeared $50 \mathrm{~ms}$ before the startle stimulus. The trial types were presented in random order with intertrial interval range from 10 to 30 s. Percent PPI of the startle response was calculated as the following formula: [ $1-$ (startle response to prepulse + startle/startle response to startle only) $] \times 100$. 
2.5.3. Social Approach-Avoidance. The test was carried out as previously described [16]. Approach-avoidance behaviors toward an unfamiliar social partner were recorded by an infrared tracking system. The arena was a plastic open field $(42 \times 42 \mathrm{~cm})$ containing an empty wire mesh cage $(10 \times 8 \mathrm{~cm})$ located at one side of the field. During the first session ("no target"), the experimental mouse was introduced into the field and its trajectory was tracked for 5 mins. During the second session ("target 1"), the conditions were identical except that a social target animal (an unfamiliar C57BL/6J male mouse) had been introduced into the cage, and for the third session ("target 2"), the social target mouse was an unfamiliar C57BL/6J female. The tracking data from both the "no target" and "target" conditions were used to determine the time spent by the experimental mouse in the "interaction zone" (an $8 \mathrm{~cm}$ wide corridor surrounding the cage) and in the "corners" of the open field opposite to the location of the cage.

2.5.4. Hole-Board Test. The apparatus was a white wooden board $(25 \times 25 \mathrm{~cm})$ with 16 evenly spaced holes. The number of head-dips was recorded by KS motor infrared monitor system over a 30 -minute period.

2.5.5. Nest Building Assay. The nest building test was performed as previously described [17]. Briefly, one square piece of material made of cotton fibre $(5 \times 5 \mathrm{~cm})$ was put in a cage with an individual mouse. Pictures of the nests were taken $16 \mathrm{~h}$ later. The quality of the nest was assessed using the following score: 1 , nest not noticeably touched; 2 , nest partially torn up; 3 , mostly shredded but not identifiable nest site; 4 , an identifiable but flat nest; 5 , a well-defined nest with walls.

2.5.6. Passive Avoidance Task. The apparatus employed in the passive avoidance task is composed of compartment shuttle chambers, one dark with shock generator and other illuminated compartments. In acquisition trials (2 days), the mouse was allowed to explore the apparatus freely for 5 mins. It would encounter an electric shock $(0.5 \mathrm{~mA}, 2 \mathrm{~s}$ duration) once it was inside of the dark chamber with all four paws. On the third day of the trial, the mouse was positioned in the illuminated compartment. Its latency to enter the dark compartment (step-through latency) was recorded automatically.

2.6. In Vivo Brain Microdialysis. In vivo brain microdialysis was carried out to measure glutamate content in the extracellular fluid in the cerebral cortex as previously described [18]. After mice had been anesthetized with inhaled isofluorane (3\%), the cerebral cortex was surgically exposed and a microdialysis probe (MAB6.14.2) was inserted into the following coordinates relative to the bregma in $\mathrm{mm}:-2$ to the anterior/posterior axis, \pm 2.0 to the lateral/medial axis, and -2.5 to the dorsal/ventral axis. Microdialysis was performed by perfusing of the probe with artificial cerebrospinal fluid at a flow rate of $2 \mu \mathrm{L} / \mathrm{min}$ via a microinfusing pump. The total volume of each dialysate sample (20 mins) was $40 \mu \mathrm{L}$. Samples were stored at $-80^{\circ} \mathrm{C}$ until use.
2.7. Preparation of Tissue Samples for HPLC. Cerebral cortex and hippocampus tissues were isolated from the brains of SNAP-25 CKO mice and their age- and sex-paired control littermates. After weighing, the samples were homogenized in ice-cold $0.4 \mathrm{M} \mathrm{HClO}_{4}$ and centrifuged at $10,000 \mathrm{~g}$ for 15 mins at $4^{\circ} \mathrm{C}$. Then, $1 \mu \mathrm{L}$ of supernatant was mixed with $750 \mu \mathrm{L}$ of $2 \mathrm{M} \mathrm{KHCO}_{3}$ and centrifuged at $3,000 \mathrm{~g}$ for 5 mins. Supernatant was gathered and stored at $-80^{\circ} \mathrm{C}$ until use.

2.8. High-Performance Liquid Chromatography (HPLC). HPLC analyses were performed in the State Key Laboratory of Medical Neurobiology of Fudan University as previously described [19]. The Agilent 1260 series neurotransmitter analyzer (Agilent Technologies, Santa Clara, CA) was utilized to detect the concentrations of amino acid neurotransmitters. Peaks and relative concentrations were identified by comparison to known external standards (Sigma-Aldrich).

2.9. Drug Treatments. Clozapine and LY354740 were purchased from Sigma-Aldrich (St. Louis, MO, USA), and lamotrigine was the product of Glaxosmithkline (Brentford, Middlesex, UK). For stock solutions, clozapine was dissolved in $0.1 \mathrm{M} \mathrm{HCl}$ and buffered with $\mathrm{NaOH}$ to achieve a final $\mathrm{pH}$ of 6.5-7.5. Riluzole was suspended in $10 w / v \%$ cyclodextrin/ saline, and LY354740 or lamotrigine was dissolved in saline. Vehicle was developed in an identical manner without the addition of drug, respectively. Concentrated aliquots of both drugs and vehicles were stored at $-20^{\circ} \mathrm{C}$. On the day of dosing, aliquots were thawed and diluted to their final concentration in sterile saline. Vehicles or clozapine $(2.5 \mathrm{mg} / \mathrm{kg})$, riluzole $(10 \mathrm{mg} / \mathrm{kg})$, and LY354740 $(15 \mathrm{mg} / \mathrm{kg})$ were injected intraperitoneally into age-matched male mice (8-12 weeks old), respectively, and submitted to the open-field test 30 mins later. Lamotrigine was administered to mice by gavage at a dose of $60 \mathrm{mg} / \mathrm{kg}$ per day for 2 weeks, followed by behavior testing.

The dose of drug was selected according to previously used doses in mouse behavioral studies [20-23] and our preliminary tests.

2.10. Statistical Analysis. Results are shown as the mean \pm SEM. Student's $t$-test was utilized to compare two means and two-way ANOVA followed by Bonferroni test to compare multiple means. The nest building scores were treated as nonparametric data, and statistical analysis was performed using Kruskal-Wallis one-way analysis on ranks followed by multiple comparison using Dunn's method. All statistical analyses were performed using Excel 2010 (Microsoft) or GraphPad Prism 5.0. $P<0.05$ was examined statistically significant.

\section{Results}

3.1. Generation of SNAP-25 Forebrain-Specific KO Mice. We generated the SNAP-25-floxed mouse strain SNAP-25 ${ }^{\text {L2/L2 }}$ through inserting of loxP cassettes in the flank sequence of exon4 loci, which caused a frame shifting by Cre-loxP recombinant mechanism (Figure 1(a)). The mouse strain was crossed with CaMKII $\alpha$-Cre transgenic mice to generate forebrain-specific SNAP-25 cKO (SNAP-25 ${ }^{\mathrm{L} 2 / \mathrm{L} 2}$ : 


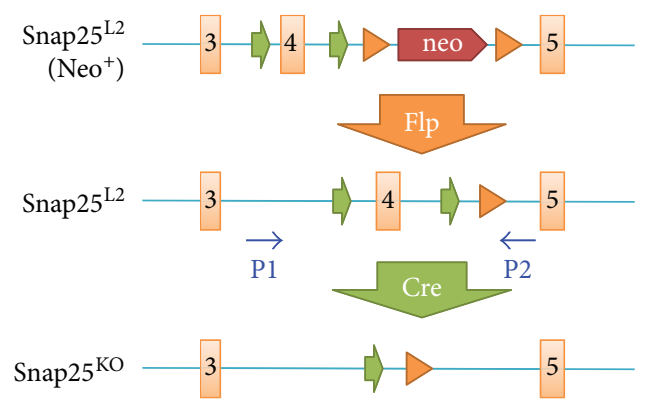

(a)

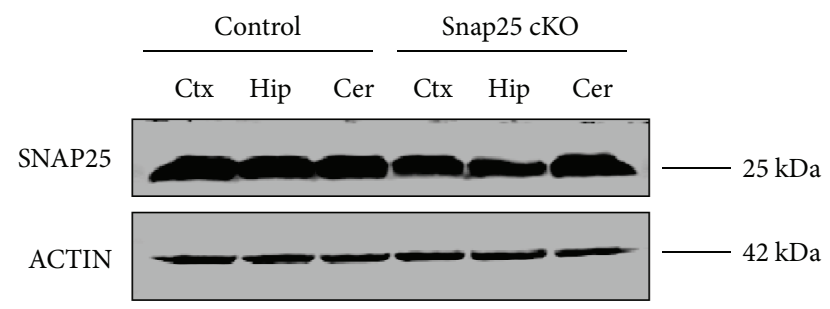

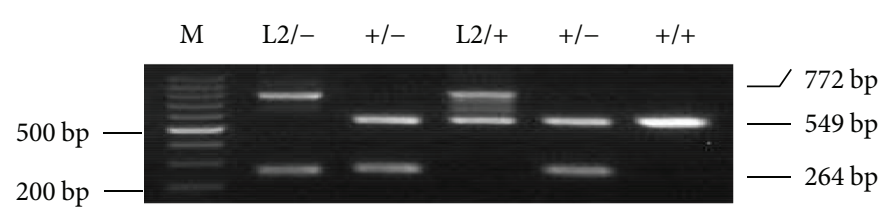

(b)

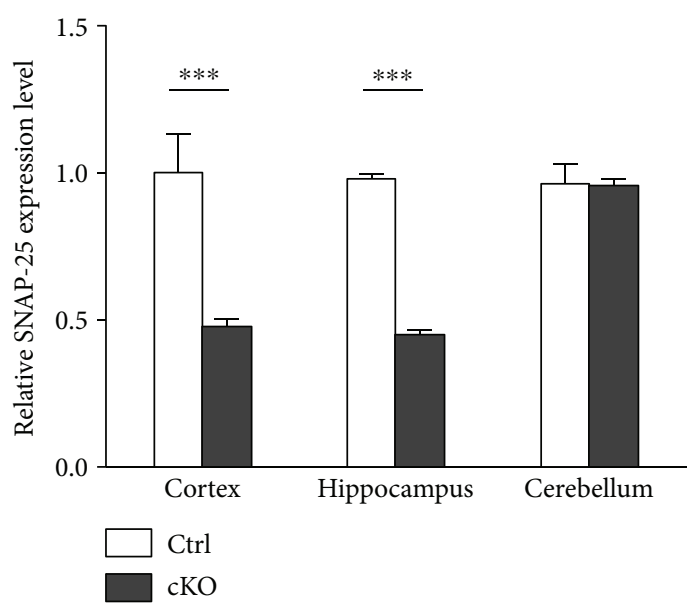

(c)

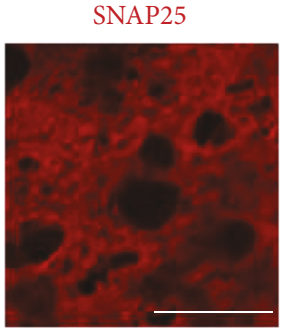

(f)

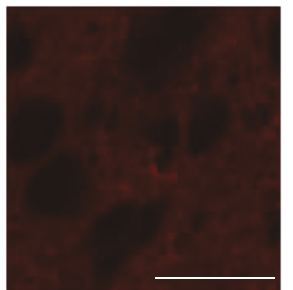

(j)

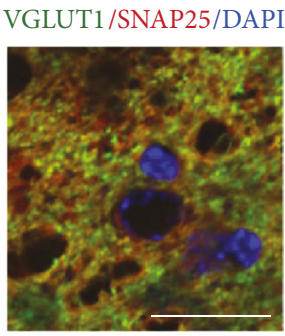

$(\mathrm{g})$

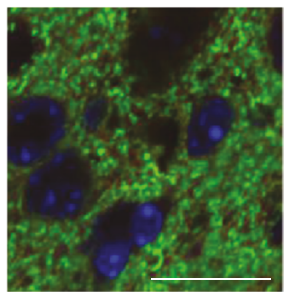

(k)

FIgURe 1: Generation of SNAP-25 forebrain-specific KO mice. (a) Targeting strategy used for the deletion of the SNAP-25 exon 4 . P1-2 referred to PCR primers for genotyping, which is located on intron flank exon 4 separately. (b) PCR genotyping of recombinant SNAP-25 locus. (c) Western blot analysis of brain extracts of Ctrl and mutant mice. Right panel: quantitative analysis of western blot images. $n=3$ per group. ${ }^{* * *} P<0.001$ compared with control littermates. (d-k) Immunostaining with anti-SNAP-25 (red) and anti-VGLUT1 (green) of cortex of sagittal sections from adult mice brains. Scale bars are $20 \mu \mathrm{m}$ in (d) and $200 \mu \mathrm{m}$ in (e).

CaMKII $\alpha$-Cre ${ }^{+/ w t}$ ) model. As we expected, the PCR product of wild-type SNAP-25 allele was $549 \mathrm{bp}$, whereas the floxed SNAP-25 allele (L2) was 772 bp and the knockout one (L-) was $264 \mathrm{bp}$. The accuracy of fragments was verified by sequencing (Figure 1(b)). SNAP-25 deletion in different brain areas was confirmed at protein levels. Dramatic reduction of SNAP-25 expression was observed in the cortex and hippocampus but no obvious change in the cerebellum of cKO mice (Figure 1(c)). Furthermore, we executed immunofluorescence examination with anti-SNAP-25 and antiVGLUT1 (glutamatergic neuron marker) staining. It was found that abundant SNAP-25-positive glutamatergic 


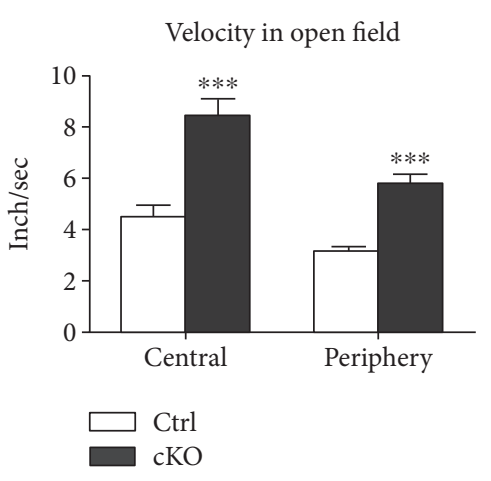

(a)

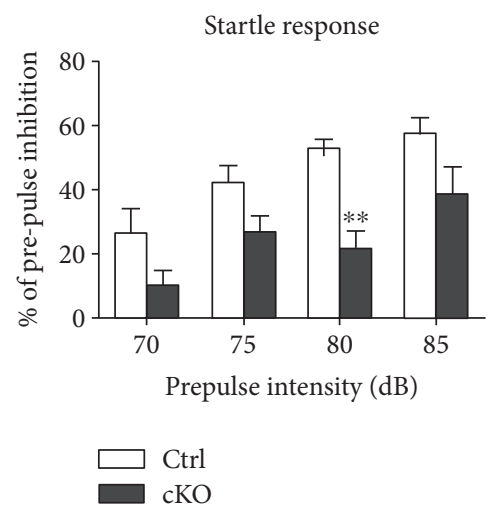

(d)

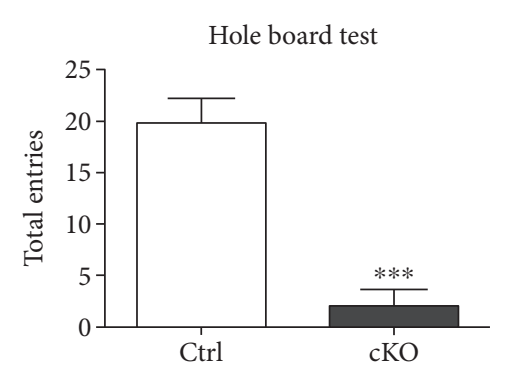

(f)

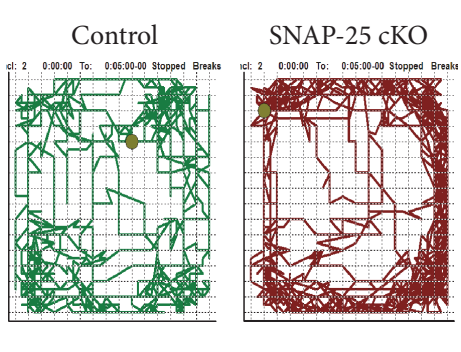

(b)

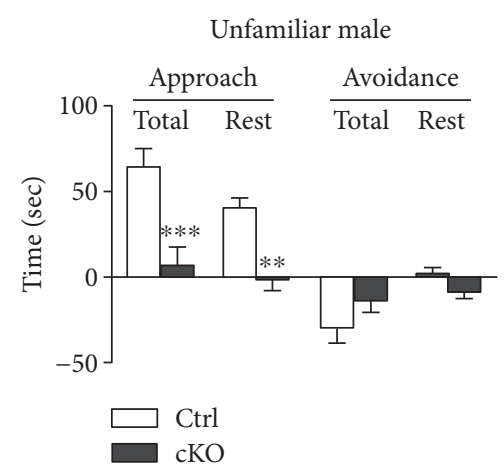

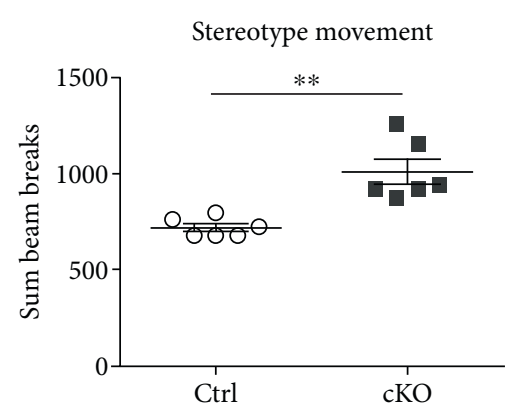

(c)

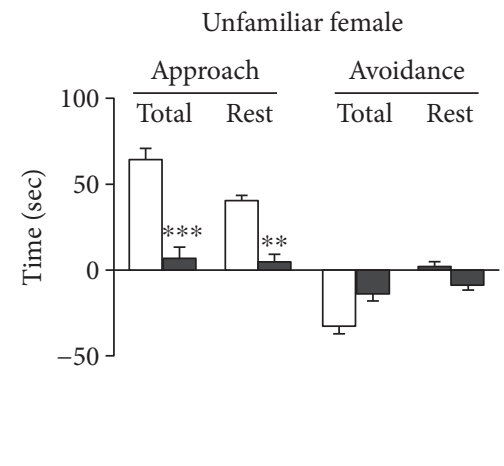

(e)

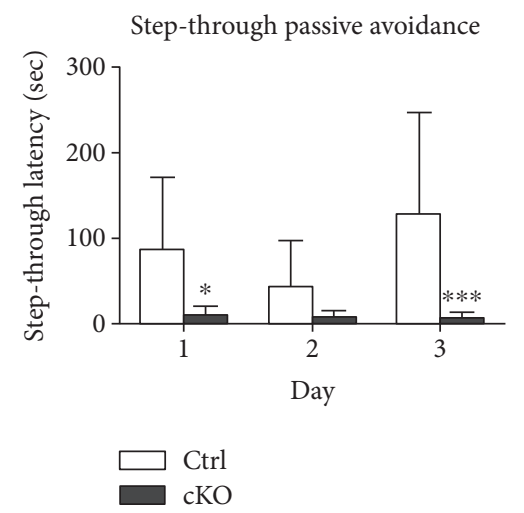

(h)

Figure 2: SNAP-25 cKO mice exhibit schizophrenia-like phenotype. (a-d) Summary plots of velocity, movement trajectory, stereotype movement in the open-field test $(n=6)$, and reduced prepulse inhibition $(n=5)$. (e) cKOs display deficient social skills as shown by social approach-avoidance test $(n=10)$. (f) cKOs occupy an impaired tendency to explore novel environment in hole-board test $(n=6)$. (g) Nest building. Left panel: pictures show the results of nesting of different genotypic mice. Right panel: statistical results of nesting scores $(n=5)$. (h) cKOs display impaired learning and memory in the step-through passive avoidance task $(n=10) .{ }^{*} P<0.05,{ }^{* *} P<0.01$, and ${ }^{* * *} P<0.001$ compared with control littermates.

neurons were detected in the cerebral cortex of Ctrl mice, whereas little staining was found in those of cKOs, confirming that SNAP-25 was inactivated in forebrain glutamatergic neurons (Figures 1(d), 1(e), 1(f), 1(g), 1(h), 1(i), $1(\mathrm{j})$, and $1(\mathrm{k}))$.

3.2. SNAP-25 cKO Mice Exhibit SZ-Like Phenotype. To determine whether SNAP-25 cKO mice occupy behavioral impairments, we subjected these animals to a battery of behavioral tests. First, in the open-field test, cKO mice showed a significant increased locomotion (3.326 \pm 0.160 versus $5.879 \pm 0.334 \mathrm{inch} / \mathrm{sec}, P<0.0001, n=6)$ and remarkably enhanced stereotype movements $(718.50 \pm 20.74$ versus $1012.00 \pm 64.42$ breaks, $P<0.05, n=6)$ compared with their control littermates (SNAP-25 ${ }^{\mathrm{L} 2 / \mathrm{L} 2}$, Ctrl, hereafter), demonstrating the abnormal hyperactivity and stereotypical behavior of cKOs (Figures 2(a), 2(b), and 2(c)). Acoustic startle test revealed that prepulse inhibition (PPI) was 
TABLE 1: Mating and nursing test of SNAP-25 cKO females (crossed with C57BL/6 J male).

\begin{tabular}{lcccccc}
\hline Genotype & Total pairs & Pregnant females & Born pups & Mean \pm SEM & Survival pups & Mean \pm SEM \\
\hline Ctrl & 10 & 9 & 57 & $5.70 \pm 0.76$ & 57 & $5.70 \pm 0.76$ \\
cKO & 10 & 4 & 25 & $2.50 \pm 1.05^{*}$ & 13 & $1.30 \pm 0.87^{* *}$ \\
\hline
\end{tabular}

${ }^{*} P<0.05$ and ${ }^{* *} P<0.01$ compared with Ctrl.

significantly decreased in the group of cKOs compared to Ctrls $[\mathrm{F}(1,8)=24.37, P<0.0001, n=5]$, and Bonferroni's post hoc comparison showed a significant disruption of PPI at the prepulse level of $80 \mathrm{~dB}(P<0.01)$ (Figure 2(d)). There was no significant difference in the startle response between cKOs and Ctrls, suggesting no apparent hearing deficit (data not shown). Thus, hyperactivity, enhanced stereotypical movements and reduced PPI of SNAP-25 cKO mice fit into the positive symptoms of SZ.

For social behavior judgement, first, we used the social approach-avoidance test to probe animals for their voluntary initiation of social interaction. When presented with an unfamiliar partner, Ctrls had the tendency to spend more time interacting socially, but cKOs displayed intense aversive responses and spent less time in close proximity to the stranger (Figure 2(e)). Also, the significantly reduced head dipping times of cKOs in the hole-board test $(19.83 \pm 2.39$ versus $1.67 \pm 1.31$ entries, $P<0.0001, n=6$ ) also reflected an impaired tendency to explore a novel environment (Figure 2(f)). By mating two genotypic females with wildtype C57BL/6J males, paired for a 4-month period, we observed that both the pregnancy rate and survival pups of cKO females were significantly lower than Ctrls, but the litter size per pregnant mother showed no difference between two groups (6.33 \pm 0.47 versus $6.25 \pm 0.63$ pups/mother, $P=0.92$, Ctrls $n=9$, and cKOs $n=4$ ), which indicates that both mating and maternal nursing behaviors were defective in cKOs (Table 1). While Ctrls could build clean and typical nests after $16 \mathrm{~h}$ with the nesting material, the nests of cKO mice were poorly formed. Substantially decreased nesting score of cKO mice demonstrated their impaired self-care ability (Figure 2(g)). Collectively, the results of the above behavioral tests showed that impaired social skills, exploratory tendency, self-care, and nursing abilities have occurred in cKOs. The cKO mice thus fit the criteria established for negative symptoms of SZ.

To determine whether cKOs have deficits in hippocampus-dependent learning and memory processes, we subjected mice to the step-through passive avoidance task. During the 3-day experiment, cKOs stepped faster into the darker-shock chamber than Ctrls. After subjected to electric shock, the step-through latencies of cKOs were more statistically pronounced compared with Ctrls, which indicated impaired learning and memory of cKOs (Figure 2(h)).

3.3. Elevated Glutamate Level in the Cortex of SNAP- 25 cKO Mice. We measured the content of glutamate in cerebral cortex and hippocampus of two mice groups by combining in vivo microdialysis and HPLC. As indicated in Figure 3(a), a significant increase in glutamate concentration was detected in the microdialysis fluid of the cerebral cortex
$(0.27 \pm 0.02$ versus $0.72 \pm 0.14 \mu \mathrm{g} / \mathrm{mL}, P<0.05$, Ctrls $n=5$, and cKOs $n=6$ ), while unchanged level were inspected in hippocampus area $(0.48 \pm 0.12$ versus $0.58 \pm 0.17 \mu \mathrm{g} / \mathrm{mL}$, $P=0.63$, Ctrls $n=5$, and cKOs $n=6$ ) in cKOs compared with Ctrl mice. However, there was no observable alteration between the concentration of amino acid neurotransmitters of homogenates freshly prepared from the same brain subregions of the cKO and Ctrl mice (Figure 3(b)).

Subsequently, by using TUBULIN and Na/K ATPase as loading controls, respectively, we examined the expression level of all three SNARE members in the cytoplasm and cell membrane fraction of the cerebral cortex. Compared with Ctrls, SNAP-25 was dramatically reduced around $60 \%$ both in cytoplasm and membrane fractions of cKOs, while the other two core members of SNARE complex: Syntaxin-1 (increased $\sim 80 \%$ ) and Vamp2 (increased $~ 96 \%$ ) were significantly increased in cell membrane part (Figure 4). There was no difference in expression of SNAP-25 homologous molecule-SNAP-23 or another important SNARE member-Munc-18 in the cell membrane of the cerebral cortex between cKOs and Ctrls. However, the expression of presynaptic calcium sensor protein-phosphorylated synaptotagmin-1-was significantly elevated about $93 \%$ in the cell membrane of the cerebral cortex of cKOs.

\subsection{Antipsychotic Drugs Attenuated Locomotor Hyperactivity} Deficits in cKO Mice. Antipsychotic drugs, clozapine (atypical schizophrenic drug), lamotrigine (broad-spectrum antiepileptic drug), LY354740 (metabotropic glutamate 2/3 receptor agonist), and riluzole (glutamate release inhibitor) were selected to examine their effects on the locomotor hyperactivities and stereotype behavior of cKO mice. Compared with controls, LY354740 treatment has no detectable effects on all four test index, while lamotrigine could reduce the stereotype movement of cKOs. Administration of either clozapine or riluzole was able to significantly attenuate the hyperactivity and stereotype movements of the SNAP-25 cKO mice (Figure 5).

\section{Discussion}

SNAP-25 is a key molecule involved in synaptic vesicle docking and neurotransmitter release. In line with its central role in neuronal function, it is thought that SNAP-25 is related to human neurological syndromes, especially SZ. In this study, we specifically deleted SNAP-25 gene in forebrain glutamatergic neurons with utilization of the Cre/LoxP strategy. The phenotypes observed in this model fit into SZ-like behaviors, which include positive symptoms (such as hyperlocomotion and reduced PPI), negative symptoms (decreased motivation and impaired social skills), and 


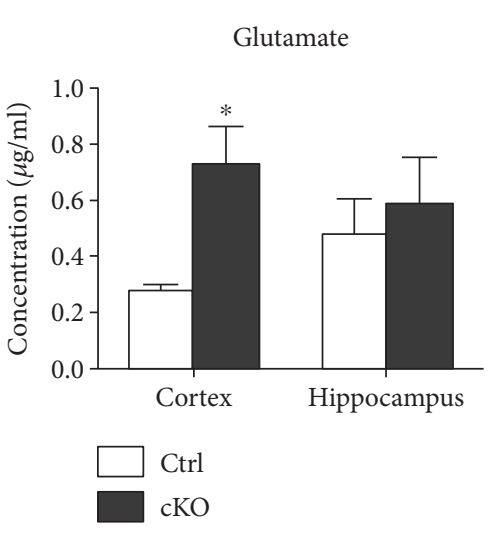

(a)

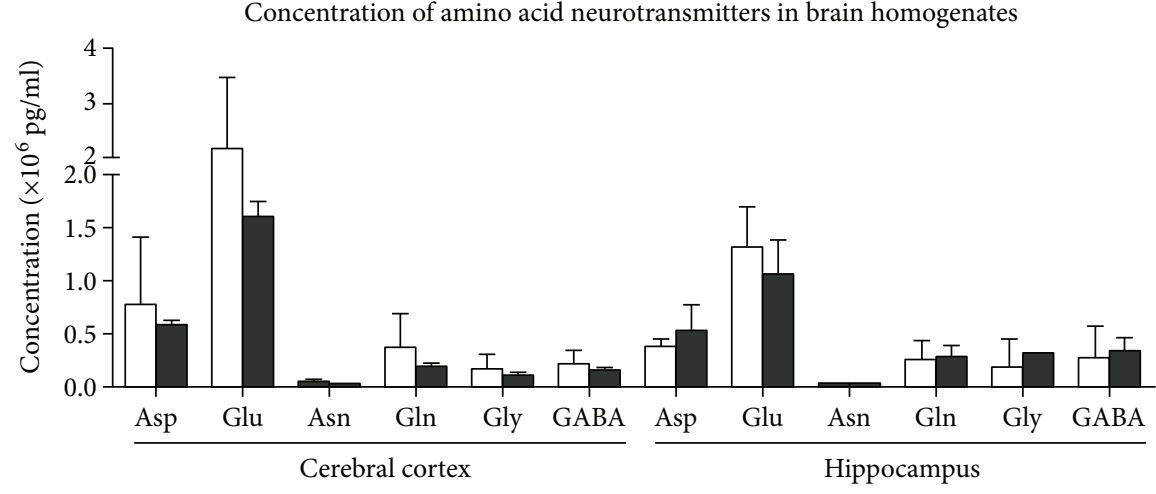

(b)

Ctrl
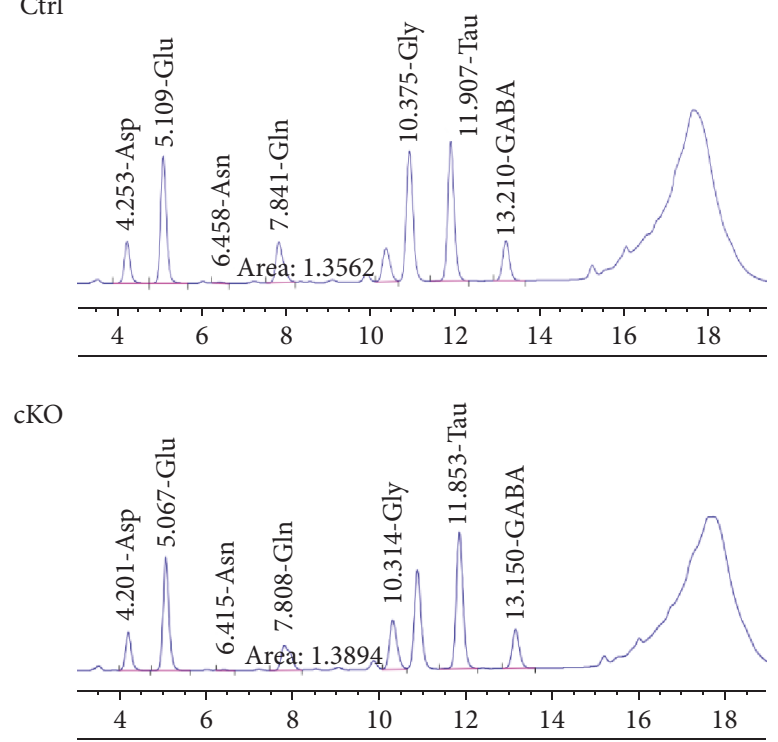

(c)

Figure 3: Elevated cortex glutamate level of SNAP-25 cKO mice. (a) The concentration of glutamate $(\mu \mathrm{g} / \mathrm{mL})$ in the microdialysis fluid (Ctrls, $n=5$; cKOs, $n=6$ ). (b) The concentration of amino acid neurotransmitters $\left(\times 10^{6} \mathrm{pg} / \mathrm{mL}\right)$ in brain homogenates $(n=5$ per group). (c) The original representative HPLC figures of different genotypic mice. ${ }^{*} P<0.05$ compared with control littermates. Asp: aspartate; Glu: glutamate; Asn: asparaginate; Gln: glutamine; Gly: glycine; GABA: $\gamma$-aminobutyric acid.

memory deficit. Our results provided in vivo functional evidence to support that altered SNAP-25 expression in the forebrain glutamatergic neurons lead to a greater effect of the illness, confirming the strong association between SNAP-25 and SZ.

It is well known that SNAP-25 plays a key role in medicating neurotransmitter release. Previous studies provided evidence that botulinum neurotoxin type A (BoNT/A) could block synaptic vesicle neuroexocytosis by proteolytic cleavage of SNAP-25, indicating that SNAP-25-deficiency could inhibit neurotransmitter release [24]. However, by in vivo brain microdialysis, we found the remarkable elevation of extracellular glutamate levels in cerebral cortex of SNAP-25 cKO mice. No noticeable difference in the total content of amino acid neurotransmitters in the same brain subregions was found between the two groups. Subsequent western blot test revealed the elevated gathering of the SNARE proteins on the cell membrane, which indicated the possibility of increased synaptic vesicle assembly and release. SNAP-25 inactivation seemed not only to fail to block synaptic transmission but also to enhance glutamatergic neurotransmitter in the cortex of cKOs. Previously, Antonucci et al. reported that reduced SNAP-25 levels lead to enhanced evoked glutamatergic transmission in hippocampal cultures and identified that this consequence was not due to changes in a releasable pool of synaptic vesicles [25]. However, we did not detect a statistical difference in hippocampus microdialysis, and we do not know the exact reason yet. We noticed that there were several differences between our and their works: (1) the developmental state of animals (adult mice and E18 mice embryos); (2) the experimental condition (intact animal under physiological condition and in vitro cultured cell model); and (3) the detective method (in vivo brain microdialysis and whole-cell patch-clamp recording). All 

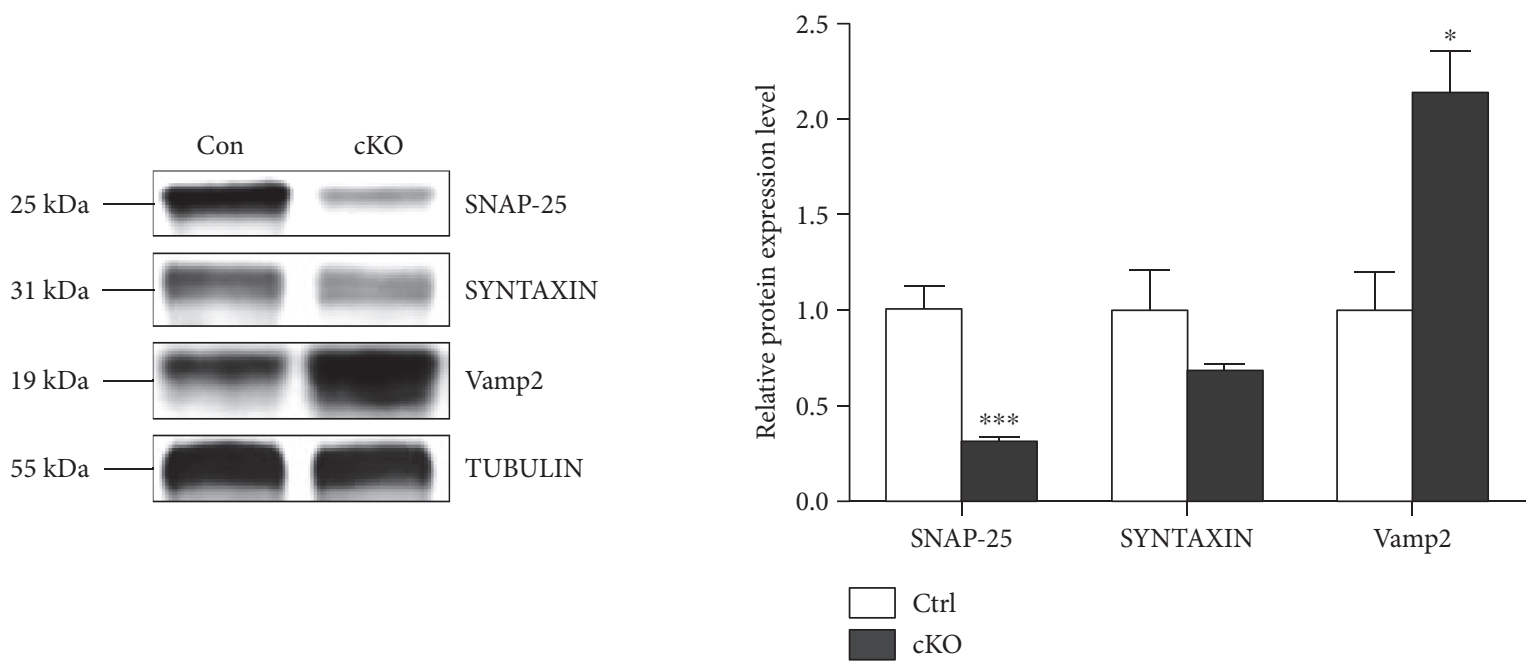

(a)
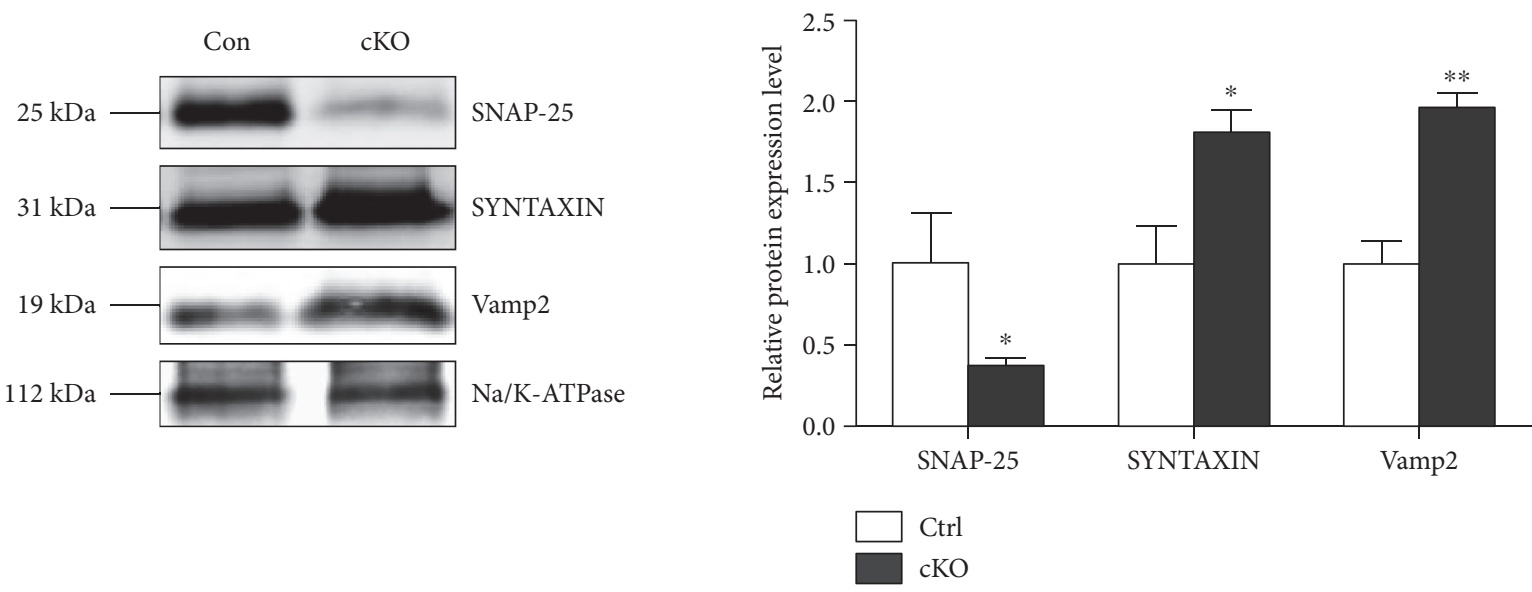

(b)
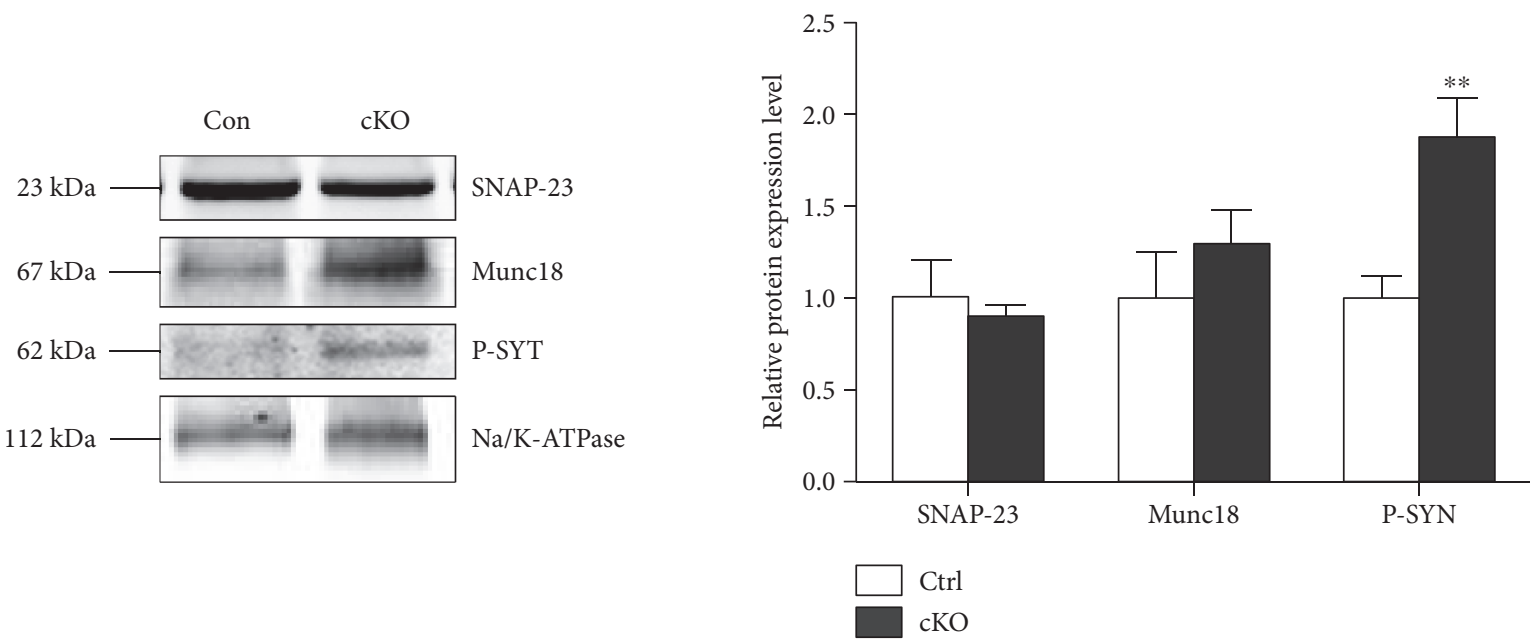

(c)

FIGURE 4: Alteration of expression pattern of SNARE-related proteins. Representative western blot (left) and densitometric analysis (right) of proteins in the cytosolic (a) and membrane (b and c) fractions prepared from mouse cerebral cortex $\left(n=3\right.$ per group). ${ }^{*} P<0.05,{ }^{* *} P<0.01$, and ${ }^{* * *} P<0.001$ compared with control littermates. P-SYT: phosphorylated synaptotagmin- 1. 


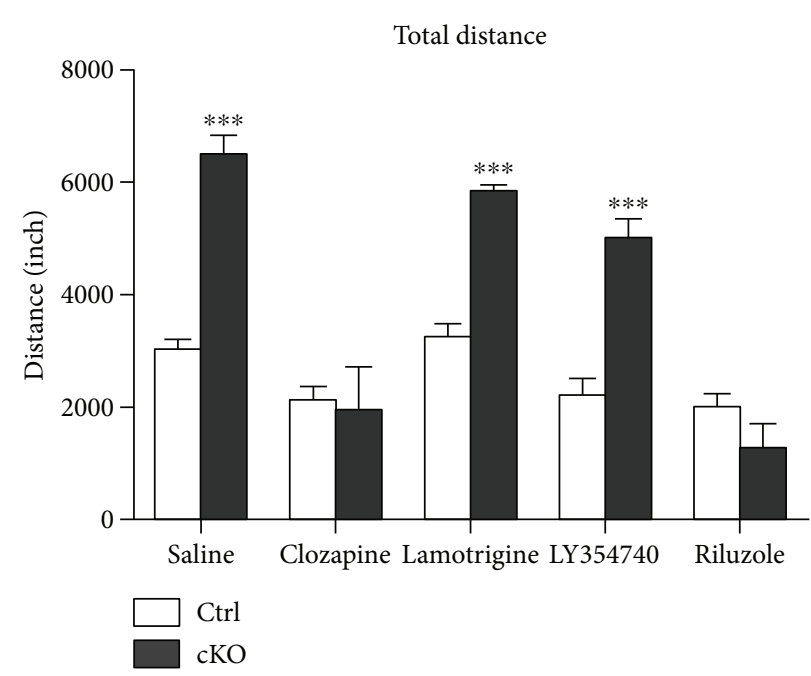

(a)

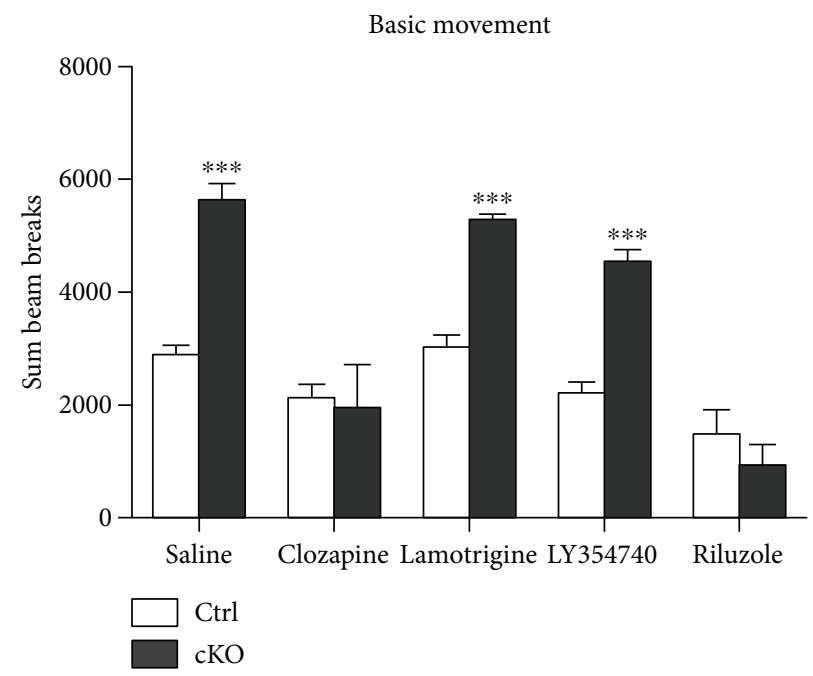

(c)

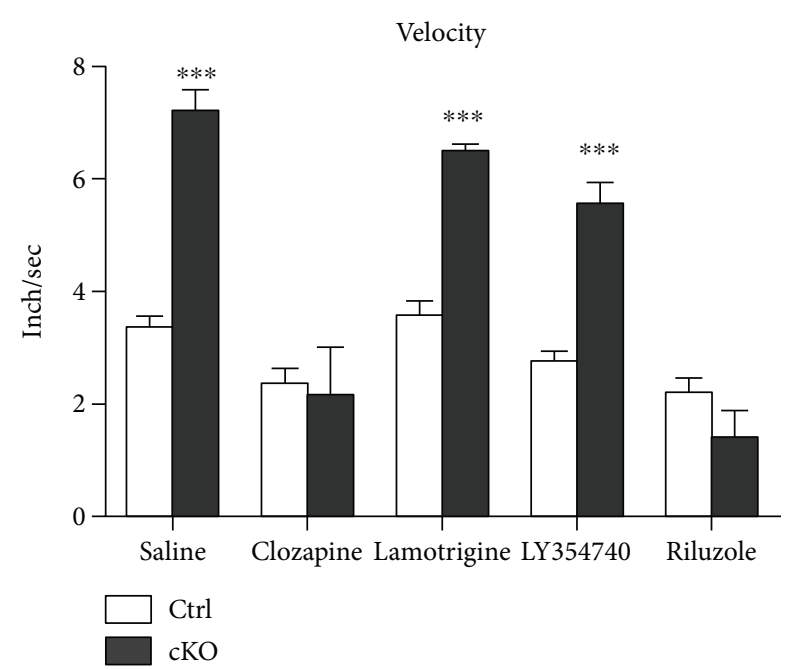

(b)

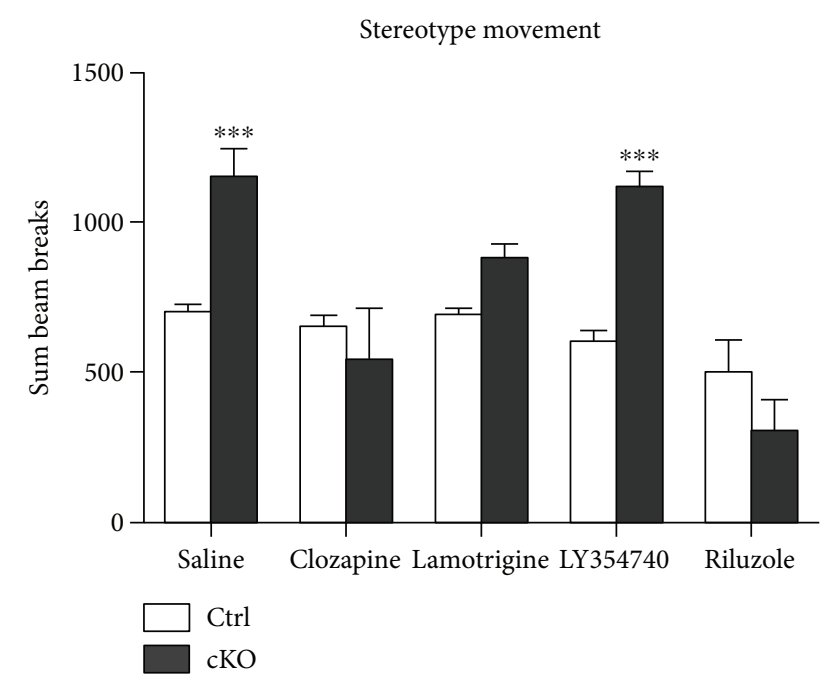

(d)

Figure 5: Antipsychotic drugs could attenuate the locomotor hyperactivity deficits in cKO mice. Summary plots of total distance (a), activity velocity (b), basic (c), and stereotype (d) movement in the open-field test. ${ }^{* * *} P<0.001$ compared with control littermates. Ctrls: $n=5$; cKOs: $n=8$.

above factors may contribute to the inconsistent results we have made.

Thus, the emerging question is how synaptic exocytosis could be enhanced without SNAP-25. To investigate the intrinsic mechanism of this phenomenon, we detected the expression level of three molecules, which are functionally related to SNAP-25 closely. These were (1) SNAP-23, the closest homolog of SNAP-25, which may be the substitution for SNAP-25 to mediate synaptic vesicle fusion [26]; (2) mammalian uncoordinated-18 (Munc-18), which has been found to have dual binding ability to syntaxin-1 and Vamp2, classified as the fourth crucial member of SNARE-pin assembly and may be another alternative for mediating neurotransmitter release $[27,28]$; and (3) as the synaptic vesicle $\mathrm{Ca}^{2+}$ sensor, synaptotagmin-1 could be phosphorylated with calcium influx and trigger the synaptic release subsequently. Endogenous SNAP-25 negatively modulates neuronal voltage-gated calcium channels (VGCCs) [11,29]. Therefore,
SNAP-25 deficiency may release VGCC activity from SNAP25 -mediated inhibition, thus resulting in exaggerated calcium influx and triggering exocytic release of glutamate. Accordingly, our molecular detection results showed the phosphorylated synaptotagmin-1 was obviously elevated, which indicated the active of VGCCs and enhanced calcium influx that may lead to greater synaptic release at the presynaptic terminal.

Moreover, we assessed the effects of commonly used antipsychotic drugs on the locomotor hyperactivities and stereotype behavior of $\mathrm{cKO}$ mice. The atypical antipsychotic clozapine can bind to receptors of serotonin, dopaminergic, and glutamatergic system. Multitarget actions make clozapine one of the most efficacious antipsychotics. It is therefore considered the "gold standard" for the treatment of SZ [30]. In regard to the cortical hyperglutamatergic state within our model, we chose three drugs that aim to inhibit presynaptic glutamate release through different pathways. These 
were (1) LY354740, a presynaptic metabotropic glutamate receptor $2 / 3$ (mGlu2/3) receptor agonist, which helps suppress the release of neurotransmitters, including glutamate and GABA [31]; (2) lamotrigine, which acts primarily through inhibition of glutamate release via blockade of voltage-sensitive sodium channels and stabilization of neuronal membrane [32]; and (3) riluzole, which has diverse effects on multiple components of the glutamatergic system, such as inhibition of glutamate release by depression of voltage-gated ion channels (sodium, potassium, and calcium) and inhibition of autoreceptors. Riluzole also affects glial cells by increasing glutamate uptake, trafficks with AMPA receptors, and so on $[33,34]$. Subsequent open-field test results showed that (1) clozapine could attenuate heightened locomotor activity of $\mathrm{cKO}$ mice. Its validity illustrated that SNAP-25 cKO mice could respond effectively to antipsychotics drug, which is the qualifying standard for an animal disease model; (2) glutamate release inhibitors occupied different efficacies on our mice and administration of riluzole has significantly corrected the hyperactivity of the SNAP-25 cKOs, whereas lamotrigine could only alleviate the stereotype movements of the mouse model, and LY354740 did not alter the activity at all. These results suggested that the hyperglutamatergic phenotype of our model may be associated closely with enhanced calcium influx rather than impaired mGlu2/3 receptor function, which corresponded with our previous observation. Further investigations are still required to provide more evidences to explore the detailed mechanisms of elevated extracellular glutamate tones in SNAP25 cKO mice.

The involvement of glutamatergic mechanism in SZ has been hypothesized for many years. SZ-relative abnormalities have been well documented in mice with mutations in postsynaptic components of glutamatergic transmission, such as NMDAR [35, 36], glycine transporter [37], and metabotropic glutamate receptor [38]. The hypofunction of postsynaptic NMDAR on inhibitory neurons that leads to disinhibition of glutamate transmission and glutamate excitotoxicity has formed the bedrock of the glutamate hypothesis of SZ. However, influence of presynaptic glutamatergic deficits is less well understood. Being a key component of glutamatergic neurotransmission at presynaptic locus, SNAP-25 deficiency induced typical SZ-like behavior demonstrated the strong association between presynaptic dysfunction and the outbreak of SZ. SNAP-25 cKO mice would be a useful novel tool for investigating presynaptic alterations that contribute to the etiopathophysiology of SZ. This research helps to consummate the pre- and postsynaptic glutamatergic pathogenesis of SZ.

\section{Conclusion}

This study showed that the forebrain glutamatergic neuronspecific SNAP-25 cKO lead to a typical SZ-like phenotype. The deficiency of SNAP-25 may lead to enhanced calcium influx and exaggerated glutamatergic release and may result in the elevated extracellular glutamate level. Riluzole attenuates the locomotor hyperactivity deficits in cKO mice. Our results provided new insight that SNAP-25 dysfunction has direct consequences on synaptic transmission and contributes to developmental of SZ. SNAP-25 cKO mouse could be a valuable new model for SZ and could be used to address questions regarding pathophysiology and etiology of the illness.

\section{Conflicts of Interest}

The authors declare that there is no conflict of interests regarding the publication of this paper.

\section{Acknowledgments}

The authors gratefully acknowledge Professor Fang Huang (The State Key Laboratory of Medical Neurobiology, Fudan University) for the technical assistance and critically reading the manuscript. This work was supported by the grant from the National Natural Science Foundation of China (no. 81271483), the Funds of Science and Technology Commission of Shanghai Municipality (nos. 14140904300 and 16DZ2280800), and the Fundamental Research Funds for the Central Universities (no. 2000219140).

\section{References}

[1] T. R. Insel, "Rethinking schizophrenia," Nature, vol. 468, no. 7321, pp. 187-193, 2010.

[2] J. Van Os, G. Kenis, and B. P. Rutten, "The environment and schizophrenia," Nature, vol. 468, no. 7321, pp. 203-212, 2010.

[3] J. Van Os and S. Kapur, "Schizophrenia," Lancet, vol. 374, no. 9690, pp. 635-645, 2009.

[4] C. M. Lewis, D. F. Levinson, L. H. Wise et al., "Genome scan meta-analysis of schizophrenia and bipolar disorder, part II: schizophrenia," American Journal of Human Genetics, vol. 73, no. 1, pp. 34-48, 2003.

[5] A. H. Fanous, M. C. Neale, B. T. Webb et al., "Novel linkage to chromosome 20p using latent classes of psychotic illness in 270 Irish high-density families," Biological Psychiatry, vol. 64, no. 2, pp. 121-127, 2008.

[6] Q. Wang, Y. Wang, W. Ji et al., "SNAP25 is associated with schizophrenia and major depressive disorder in the Han Chinese population," The Journal of Clinical Psychiatry, vol. 76, no. 1, pp. e76-e82, 2015.

[7] V. E. Barakauskas, A. Moradian, A. M. Barr et al., "Quantitative mass spectrometry reveals changes in SNAP-25 isoforms in schizophrenia," Schizophrenia Research, vol. 177, no. 1-3, pp. 44-51, 2016.

[8] C. N. Karson, R. E. Mrak, K. O. Schluterman, W. Q. Sturner, J. G. Sheng, and W. S. T. Griffin, "Alterations in synaptic proteins and their encoding mRNAs in prefrontal cortex in schizophrenia: a possible neurochemical basis for 'hypofrontality'," Molecular Psychiatry, vol. 4, no. 1, pp. 39-45, 1999.

[9] S. H. Fatemi, J. A. Earle, J. M. Stary, S. Lee, and J. Sedgewick, "Altered levels of the synaptosomal associated protein SNAP-25 in hippocampus of subjects with mood disorders and schizophrenia," Neuroreport, vol. 12, no. 15, pp. 32573262, 2001.

[10] M. Matteoli, D. Pozzi, C. Grumelli et al., "The synaptic split of SNAP-25: different roles in glutamatergic and GABAergic neurons?," Neuroscience, vol. 158, no. 1, pp. 223-230, 2009. 
[11] S. B. Condliffe, I. Corradini, D. Pozzi, C. Verderio, and M. Matteoli, "Endogenous SNAP-25 regulates native voltagegated calcium channels in glutamatergic neurons," The Journal of Biological Chemistry, vol. 285, no. 32, pp. 24968-24976, 2010.

[12] F. Antonucci, I. Corradini, G. Fossati, R. Tomasoni, E. Menna, and M. Matteoli, "SNAP-25, a known presynaptic protein with emerging postsynaptic functions," Frontiers in Synaptic Neuroscience, vol. 8, p. 7, 2016.

[13] J. Z. Tsien, D. F. Chen, D. Gerber et al., "Subregion- and cell type-restricted gene knockout in mouse brain," Cell, vol. 87, no. 7, pp. 1317-1326, 1996.

[14] M. He, Y. Liu, X. Wang, M. Q. Zhang, G. J. Hannon, and Z. J. Huang, "Cell-type-based analysis of microRNA profiles in the mouse brain," Neuron, vol. 73, no. 1, pp. 35-48, 2012.

[15] I. H. Kim, S. K. Park, S. T. Hong et al., "Inositol 1,4,5-trisphosphate 3-kinase a functions as a scaffold for synaptic Rac signaling," The Journal of Neuroscience, vol. 29, no. 44, pp. 1403914049, 2009.

[16] O. Berton, C. A. Mcclung, R. J. Dileone et al., "Essential role of BDNF in the mesolimbic dopamine pathway in social defeat stress," Science, vol. 311, no. 5762, pp. 864-868, 2006.

[17] H. Zhang, E. Kang, Y. Wang et al., "Brain-specific Crmp2 deletion leads to neuronal development deficits and behavioural impairments in mice," Nature Communications, vol. 7, 2016.

[18] Y. K. Wang, D. Shen, Q. Hao et al., "Overexpression of angiotensin-converting enzyme 2 attenuates tonically active glutamatergic input to the rostral ventrolateral medulla in hypertensive rats," American Journal of Physiology. Heart and Circulatory Physiology, vol. 307, no. 2, pp. H182-H190, 2014.

[19] M. Han, X. Xiao, Y. Yang et al., "SIP30 is required for neuropathic pain-evoked aversion in rats," The Journal of Neuroscience, vol. 34, no. 2, pp. 346-355, 2014.

[20] C. L. Schmid, J. M. Streicher, H. Y. Meltzer, and L. M. Bohn, "Clozapine acts as an agonist at serotonin $2 \mathrm{~A}$ receptors to counter MK-801-induced behaviors through a $\beta$ arrestin2independent activation of Akt," Neuropsychopharmacology, vol. 39, no. 8, pp. 1902-1913, 2014.

[21] C. Procaccini, M. Maksimovic, T. Aitta-Aho, E. R. Korpi, and A. M. Linden, "Reversal of novelty-induced hyperlocomotion and hippocampal c-Fos expression in GluA1 knockout male mice by the mGluR2/3 agonist LY354740," Neuroscience, vol. 250, pp. 189-200, 2013.

[22] M. Y. Zhang, C. Y. Zheng, M. M. Zou et al., "Lamotrigine attenuates deficits in synaptic plasticity and accumulation of amyloid plaques in APP/PS1 transgenic mice," Neurobiology of Aging, vol. 35, no. 12, pp. 2713-2725, 2014.

[23] N. Egashira, R. Okuno, S. Harada et al., "Effects of glutamate-related drugs on marble-burying behavior in mice: implications for obsessive-compulsive disorder," European Journal of Pharmacology, vol. 586, no. 1-3, pp. 164170, 2008.

[24] I. Matak and Z. Lackovic, "Botulinum neurotoxin type a: actions beyond SNAP-25?," Toxicology, vol. 335, pp. 79-84, 2015.

[25] F. Antonucci, I. Corradini, R. Morini et al., "Reduced SNAP-25 alters short-term plasticity at developing glutamatergic synapses," EMBO Reports, vol. 14, no. 7, pp. 645-651, 2013.

[26] S. Arora, I. Saarloos, R. Kooistra, R. van de Bospoort, M. Verhage, and R. F. Toonen, "SNAP-25 gene family members differentially support secretory vesicle fusion," Journal of Cell Science, vol. 130, no. 11, pp. 1877-1889, 2017.

[27] A. Pertsinidis, K. Mukherjee, M. Sharma et al., "Ultrahigh-resolution imaging reveals formation of neuronal SNARE/ Munc18 complexes in situ," Proceedings of the National Academy of Sciences of the United States of America, vol. 110, no. 30, pp. E2812-E2820, 2013.

[28] D. Parisotto, J. Malsam, A. Scheutzow, J. M. Krause, and T. H. Sollner, "SNAREpin assembly by Munc18-1 requires previous vesicle docking by synaptotagmin 1," The Journal of Biological Chemistry, vol. 287, no. 37, pp. 31041-31049, 2012.

[29] S. B. Condliffe and M. Matteoli, "Inactivation kinetics of voltage-gated calcium channels in glutamatergic neurons are influenced by SNAP-25," Channels, vol. 5, no. 4, pp. 304307, 2011.

[30] H. Y. Meltzer, "Update on typical and atypical antipsychotic drugs," Annual Review of Medicine, vol. 64, no. 1, pp. 393406, 2013.

[31] R. Farazifard and S. H. Wu, "Metabotropic glutamate receptors modulate glutamatergic and GABAergic synaptic transmission in the central nucleus of the inferior colliculus," Brain Research, vol. 1325, pp. 28-40, 2010.

[32] A. L. Meehan, X. Yang, B. D. McAdams, L. Yuan, and S. M. Rothman, "A new mechanism for antiepileptic drug action: vesicular entry may mediate the effects of levetiracetam," Journal of Neurophysiology, vol. 106, no. 3, pp. 1227-1239, 2011.

[33] N. Lamanauskas and A. Nistri, "Riluzole blocks persistent $\mathrm{Na}^{+}$ and $\mathrm{Ca}^{+}{ }^{+}$currents and modulates release of glutamate via presynaptic NMDA receptors on neonatal rat hypoglossal motoneurons in vitro," The European Journal of Neuroscience, vol. 27, no. 10, pp. 2501-2514, 2008.

[34] R. Machado-Vieira, G. Salvadore, L. A. Ibrahim, N. Diaz-Granados, and C. A. Zarate Jr, "Targeting glutamatergic signaling for the development of novel therapeutics for mood disorders," Current Pharmaceutical Design, vol. 15, no. 14, pp. 1595-1611, 2009.

[35] K. Nakazawa, V. Jeevakumar, and K. Nakao, "Spatial and temporal boundaries of NMDA receptor hypofunction leading to schizophrenia," NPJ Schizophrenia, vol. 3, no. 1, p. 7, 2017.

[36] D. Timucin, O. Ozdemir, and M. Parlak, "The role of NMDAR antibody in the etiopathogenesis of schizophrenia," Neuropsychiatric Disease and Treatment, vol. 12, pp. 2327-2332, 2016.

[37] E. Dunayevich, R. W. Buchanan, C. Y. Chen et al., "Efficacy and safety of the glycine transporter type-1 inhibitor AMG 747 for the treatment of negative symptoms associated with schizophrenia," Schizophrenia Research, vol. 182, pp. 90-97, 2017.

[38] J. Maksymetz, S. P. Moran, and P. J. Conn, "Targeting metabotropic glutamate receptors for novel treatments of schizophrenia," Molecular Brain, vol. 10, no. 1, p. 15, 2017. 

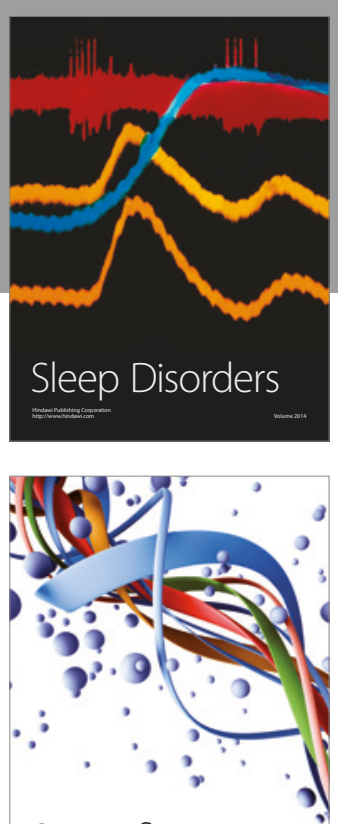

Scientifica
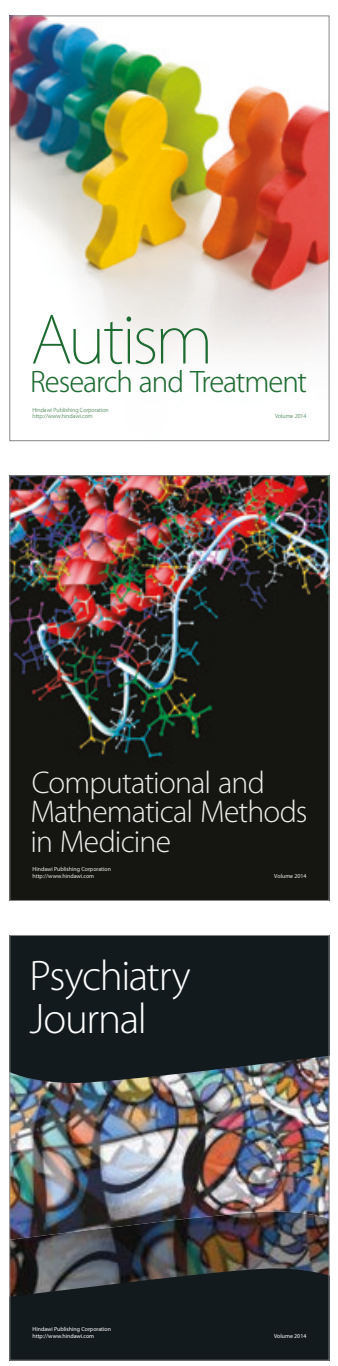
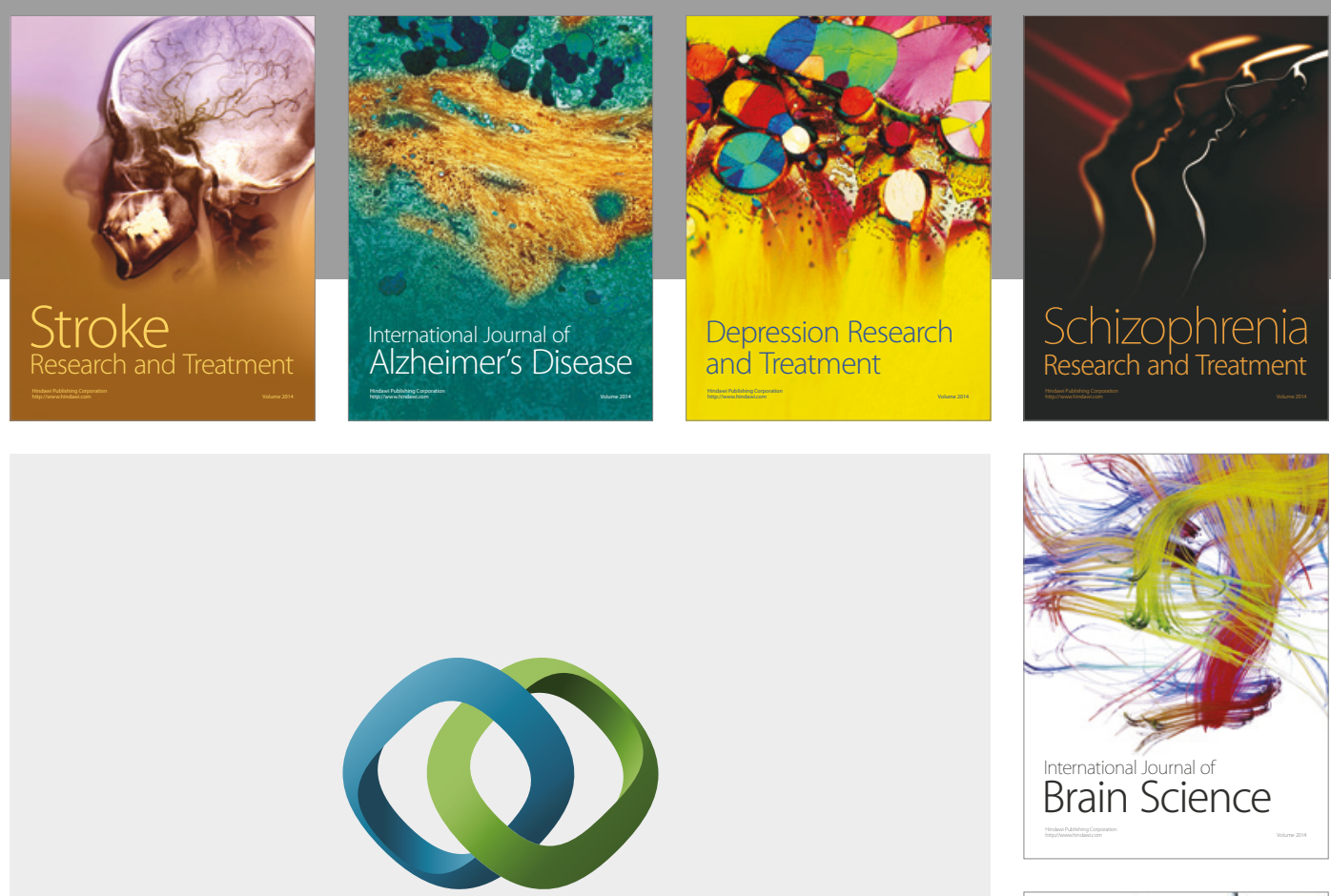

\section{Hindawi}

Submit your manuscripts at

https://www.hindawi.com
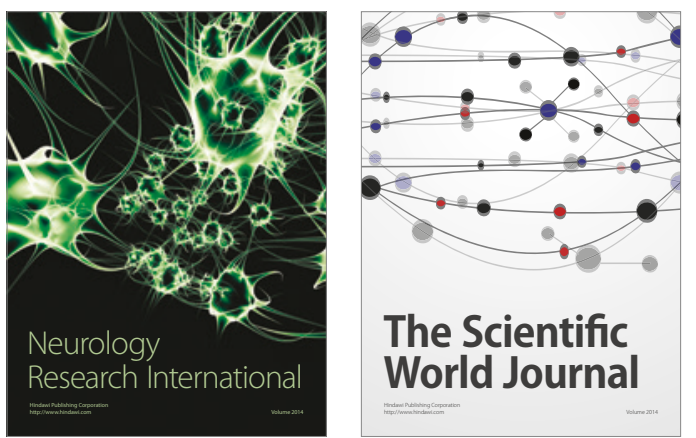

The Scientific World Journal

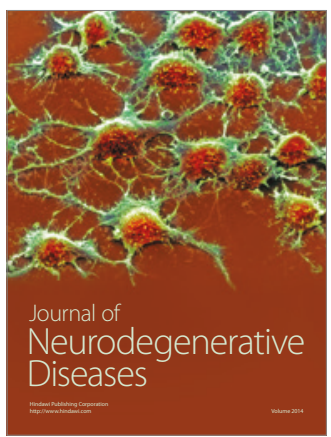

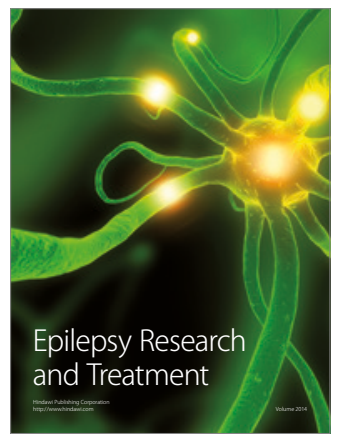

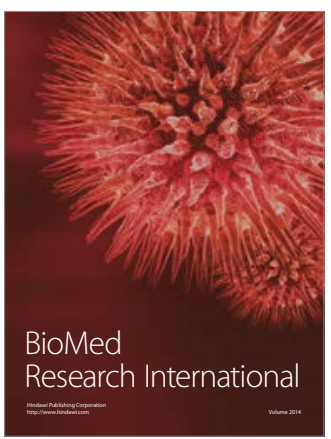

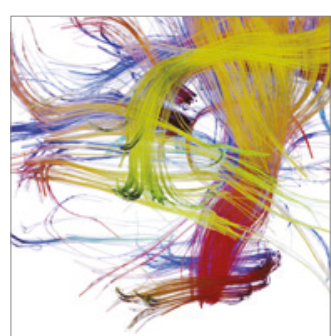

Brain Science

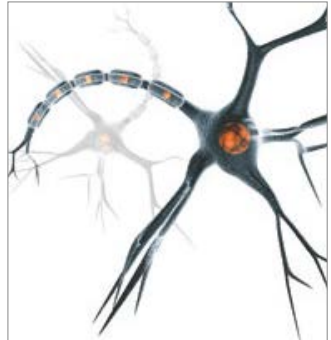

Neural Plasticity
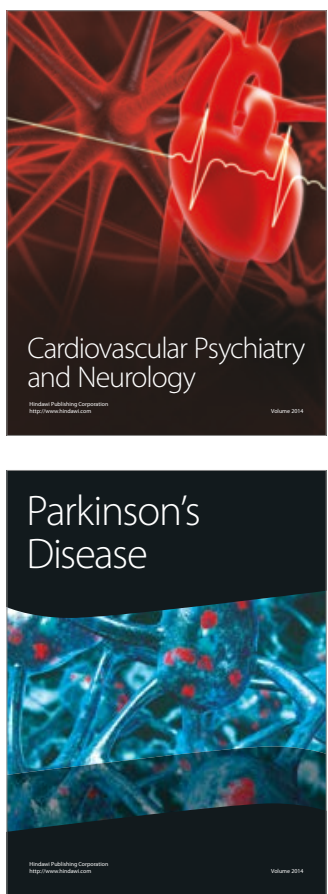